\title{
Positive diversity-functioning relationships in model communities of methanotrophic bacteria
}

\author{
Elvira Schnyder, ${ }^{1}$ Paul L. E. Bodelier, ${ }^{2}$ Martin Hartmann, ${ }^{3}$ Ruth Henneberger, ${ }^{4,6}$ and Pascal A. Niklaus ${ }^{1,5,7}$ \\ ${ }^{1}$ Department of Evolutionary Biology and Environmental Studies, University of Zürich, Winterthurerstrasse 190, CH-8057 Zurich, Switzerland \\ ${ }^{2}$ Department of Microbial Ecology, Netherlands Institute of Ecology (NIOO-KNAW), Droevendaalsesteeg 10, 6708 PB Wageningen, The Netherlands \\ ${ }^{3}$ Forest Soils and Biogeochemistry, Swiss Federal Research Institute WSL, Zürcherstrasse 111, CH-8903 Birmensdorf, Switzerland \\ ${ }^{4}$ Institute of Biogeochemistry and Pollutant Dynamics, ETH Zurich, Universitätstrasse 16, CH-8092 Zurich, Switzerland \\ ${ }^{5}$ University of Zurich Research Priority Program Global Change and Biodiversity, University of Zürich, Winterthurerstrasse 190, \\ CH-8057 Zurich, Switzerland
}

\begin{abstract}
Biodiversity enhances ecosystem functions such as biomass production and nutrient cycling. Although the majority of the terrestrial biodiversity is hidden in soils, very little is known about the importance of the diversity of microbial communities for soil functioning. Here, we tested effects of biodiversity on the functioning of methanotrophs, a specialized group of soil bacteria that plays a key role in mediating greenhouse gas emissions from soils. Using pure strains of methanotrophic bacteria, we assembled artificial communities of different diversity levels, with which we inoculated sterile soil microcosms. To assess the functioning of these communities, we measured methane oxidation by gas chromatography throughout the experiment and determined changes in community composition and community size at several time points by quantitative PCR and sequencing. We demonstrate that microbial diversity had a positive overyielding effect on methane oxidation, in particular at the beginning of the experiment. This higher assimilation of $\mathrm{CH}_{4}$ at high diversity translated into increased growth and significantly larger communities towards the end of the study. The overyielding of mixtures with respect to $\mathrm{CH}_{4}$ consumption and community size were positively correlated. The temporal $\mathrm{CH}_{4}$ consumption profiles of strain monocultures differed, raising the possibility that temporal complementarity of component strains drove the observed community-level strain richness effects; however, the community niche metric we derived from the temporal activity profiles did not explain the observed strain richness effect. The strain richness effect also was unrelated to both the phylogenetic and functional trait diversity of mixed communities. Overall, our results suggest that positive biodiversity-ecosystem-function relationships show similar patterns across different scales and may be widespread in nature. Additionally, biodiversity is probably also important in natural methanotrophic communities for the ecosystem function methane oxidation. Therefore, maintaining soil conditions that support a high diversity of methanotrophs may help to reduce the emission of the greenhouse gas methane.
\end{abstract}

Key words: biodiversity; ecosystem function; mechanisms; methane oxidation; methanotrophic bacteria; niche complementarity; overyielding; soil ecology.

\section{INTRODUCTION}

The dramatic loss of biodiversity as a consequence of anthropogenic activities has prompted many experiments that investigated whether and how ecosystem functioning depends on the diversity of the organisms present. To date, most research has focused on aboveground organisms, in particular plants. The majority of these studies have shown a positive relationship between the number of plant species and ecosystem functions such as biomass production. These effects are remarkably consistent across different ecosystem types like grasslands, forests, and marine ecosystems (Balvanera et al. 2006, Cardinale et al. 2012).

Although most studies so far have focused on aboveground organisms, in terrestrial ecosystems, the majority of biodiversity is found belowground. Soil microbial communities are extremely diverse and drive important biogeochemical processes including the decomposition of organic matter

Manuscript received 15 June 2017; revised 1 November 2017; accepted 20 December 2017. Corresponding Editor: Steven D. Allison.

${ }^{6}$ Present address: Institute of Molecular Health Sciences, ETH Zurich, Rämistrasse 101, CH-8092 Zurich, Switzerland.

${ }^{7}$ Corresponding author. E-mail: pascal.niklaus@ieu.uzh.ch and soil nutrient transformations. Soil microbes also mediate fluxes of greenhouse gases, including methane $\left(\mathrm{CH}_{4}\right)$, and therefore are important in light of climate change (Bodelier and Steenbergh 2014). Despite their fundamental significance, little is known to date about the importance of the diversity of these organisms for the functions they promote (Krause et al. 2014b). Positive biodiversity-ecosystem-functioning (BEF) relationships in communities of aboveground macroorganisms have been attributed to three main groups of mechanisms: (1) sampling effects (Aarssen 1997), also known as selection probability effects, refer to the phenomenon that more diverse communities are more likely to contain species that perform particularly well; (2) niche differentiation among species can support the complementary use of resources, thereby reducing competition and allowing for a higher community-level yield (Tilman et al. 1996, Tilman 1997); also, complementarity with respect to enemies will reduce the impact these have if their effect is strongly dependent on the density of hosts (e.g., Maron et al. 2011); (3) facilitation, i.e., positive effects on a species that are mediated by modification of environmental conditions by another species, may also promote a better functioning of mixtures. A frequent case of facilitation in biodiversity experiments is 
dinitrogen fixation by legumes (e.g., Spehn et al. 2002). It remains unclear whether and to which extent these groups of mechanisms also operate in soil microbial communities. One reason is that most investigations of BEF relationships in microbial systems to date have been observational studies along environmental gradients (Krause et al. 2014b). In such studies, correlations between diversity and functioning can emerge for many reasons including covariation of diversity with environmental parameters that modify ecosystem functioning independent of diversity. The other reason is that a detailed analyses of mechanisms often requires that a measured community-level function can be broken down into contributions of the component species, which is more challenging in microbial systems than with macroorganisms.

Studies in which microbial diversity has been manipulated directly and systematically have only recently begun to emerge (e.g., Bell et al. 2005, Replansky and Bell 2009, Bier et al. 2015, Fetzer et al. 2015, Delgado-Baquerizo et al. 2016). Many of these studies concerned relatively broad, diversified groups of microbes that drive very general functions such as soil respiration or decomposition. The microbes that carry out these functions often are taxonomically and phylogenetically very diverse and hence there may be high functional redundancy (Nannipieri et al. 2003) and the loss of diversity may, at least initially, have no or only limited consequences. Nevertheless, the few experiments available suggest that niche complementarity can, to some extent, promote community-level functioning through resource partitioning (e.g., Salles et al. 2012).

In the present study, we tested for effects of the diversity of a specialized group of bacteria, the methanotrophs, on their activity and growth. Methanotrophs are obligate aerobic bacteria that oxidize $\mathrm{CH}_{4}$ and assimilate $\mathrm{CH}_{4}-\mathrm{C}$ into biomass. They constitute the most important biotic sink for atmospheric $\mathrm{CH}_{4}$ and thus play a key role in the $\mathrm{CH}_{4}$ cycle by affecting atmospheric $\mathrm{CH}_{4}$ concentrations and the associated greenhouse effect. In wetland systems, methanotrophs together with anaerobic $\mathrm{CH}_{4}$ oxidizers often oxidize significant fractions of the $\mathrm{CH}_{4}$ produced by methanogenic Archaea, sometimes over $90 \%$, and therefore substantially reduce $\mathrm{CH}_{4}$ emissions to the atmosphere (Le Mer and Roger 2001). Based on our current knowledge, methanotrophs are spread over a limited number of phyla, with most members found in the alpha and gamma division of the Proteobacteria (Lüke and Frenzel 2011, Knief 2015). In many ecosystems, their diversity is relatively low with typically up to a few dozen OTUs found (e.g., Levine et al. 2011, Lüke and Frenzel 2011, Siljanen et al. 2011, Serrano-Silva et al. 2014, Knief 2015). The generally limited diversity and high functional specialization of methanotrophs raises the possibility of a low functional redundancy. $\mathrm{CH}_{4}$ oxidation may therefore be more vulnerable to diversity loss than is typically found for functions driven by more diverse groups of microorganisms with more versatile metabolism. Also, their methanotrophic nature makes them well suited for studying microbial BEF relationships.

Here, we used 10 pure methanotroph strains to create artificial communities composed of 1-10 strains to test for the effects of their biodiversity on their activity and growth. These communities were incubated for six weeks in microcosms with above-atmospheric headspace $\mathrm{CH}_{4}$ concentrations. Throughout the experiment, we quantified methanotrophic activity by assessing $\mathrm{CH}_{4}$ consumption by gas chromatography. Growth of the methanotrophic communities was estimated by measuring the abundance of a gene encoding for the particulate methane monooxygenase ( Finally, we determined the composition of the experimental communities by next-generation sequencing of ribosomal (16S rRNA) and functional gene (pmoA) fragments. Our objective was to test whether increased methanotroph diversity promotes $\mathrm{CH}_{4}$ consumption and the growth of methanotrophic communities. We further tested whether over-yielding of mixed communities was related to functional trait diversity and the phylogenetic relatedness of the strains combined in mixtures, and whether diversity and composition-effects on activity and growth were positively correlated, i.e., whether strain mixtures that over-yielded in terms of community size also showed particularly high $\mathrm{CH}_{4}$ consumption rates.

\section{Materials ANd Methods}

\section{Study species and experimental setup}

We created a diversity gradient using 10 strains of methanotrophic bacteria that we grew in pure culture. All aerobic methanotrophic bacteria known to date belong either to the Gammaproteobacteria (type I), Alphaproteobacteria (type II), or Verrucomicrobia (Type III; McDonald et al. 2008, Knief 2015). In our experiment, we used three Type I and seven Type II methanotrophs (Table 1). These strains occur in habitats in which methanogens are active and soil $\mathrm{CH}_{4}$ concentrations typically high. These methanotrophs exhibit a so called "low affinity" kinetic, i.e., they have a high capacity to oxidize $\mathrm{CH}_{4}$ but only at concentrations that are well above atmospheric levels (Dunfield et al. 1999). We initially grew all strains in liquid batch cultures in nitrate mineral salt medium (NMS medium; Dedysh et al. 1998). Liquid batch cultures consisted of $100-\mathrm{mL}$ serum flasks with $20 \mathrm{~mL}$ NMS medium and $20 \mathrm{~mL}$ $\mathrm{CH}_{4}$ added to the headspace. The liquid batch cultures were kept at $25^{\circ} \mathrm{C}$ (except M. capsulatus at $36^{\circ} \mathrm{C}$ and $M$. tundripaludum at $23^{\circ} \mathrm{C}$ ) until the cultures reached the stationary growth phase, then at $4^{\circ} \mathrm{C}$ until they were added to the microcosms.

We constructed microcosms from 50-mL centrifugation tubes (Sarstedt, Nümbrecht, Germany) that we pre-filled with $4 \mathrm{~g}$ dry equivalent mass of autoclaved soil from a calcareous meadow (Nenzlingen, Switzerland; 473' ${ }^{\prime}$ N, 7³ $34^{\prime}$ E, 520 m above sea level; silty clay loam soil, $41 \%$ clay, $52 \%$ silt $3.9 \% \mathrm{C}$, $0.33 \% \mathrm{~N}, \mathrm{pH} \approx 7,8$; Niklaus et al. 2003), which has a very low methanotroph abundance (relative abundance of pmo $A$ gene measured by qPCR $<0.01 \%$ of that measured in inoculated microcosms). We established a methanotroph diversity gradient $(1,2,4,6$, or 10 strains) in these microcosms by adding $1 \mathrm{~mL}$ inoculum and $1.2 \mathrm{~mL}$ NMS medium. The inoculum was prepared by mixing aliquots of the pure liquid cultures that had been diluted to approximately 5,000,000 cells $/ \mathrm{mL}$ with NMS medium based on measurements of their original cell density (CASY Model TTC cell counter, Roche Innovatis AG, Bielefeld, Germany). The amounts of pure culture 
TABLE 1. Methanotrophic strains used to assemble the artificial communities and their traits used to calculate functional diversity (FD).

\begin{tabular}{|c|c|c|c|c|c|c|c|c|c|c|}
\hline Characteristic & $\begin{array}{c}\text { M. lut }{ }^{1} \\
\text { ATCC } \\
49878 \mathrm{~T}\end{array}$ & $\begin{array}{l}\text { M. } \text { tun }^{2} \\
\text { SV96 }\end{array}$ & $\begin{array}{l}\text { M. } \text { cap }^{1} \\
\text { Bath }\end{array}$ & $\begin{array}{l}\text { M. ech } \\
\text { IMET } \\
10491\end{array}$ & $\begin{array}{l}\text { M. } \text { hir }^{3} \\
\text { CSC1 }\end{array}$ & $\begin{array}{l}\text { M. par } \\
\text { OBBP }\end{array}$ & $\begin{array}{l}\text { M. } \text { sil }^{4} \\
\text { BL2 }\end{array}$ & $\begin{array}{l}\text { M. } \operatorname{ros}^{5} \\
\text { SV97 }\end{array}$ & $\begin{array}{l}\text { M. spo }{ }^{1} \\
\text { NCIMB } \\
11126\end{array}$ & $\begin{array}{l}\text { M. } t r i^{1} \\
\text { OB } 3 \mathrm{~b}\end{array}$ \\
\hline Type & I & I & I & II & II & II & II & II & II & II \\
\hline Motility & 0 & 0 & 0 & 0 & 0 & 0 & 0 & 0 & 1 & 1 \\
\hline Can use methanol & 1 & 0 & $1^{6}$ & 1 & 1 & 1 & 0 & 1 & 1 & 1 \\
\hline $\begin{array}{l}\text { Can use other C } \\
\text { sources }\end{array}$ & 0 & 0 & 1 & 0 & 0 & 0 & 0 & 1 & 0 & 0 \\
\hline Fixes nitrogen & 0 & 1 & 1 & 1 & 1 & 1 & 1 & 1 & 1 & 1 \\
\hline Cell length $^{7}(\mu \mathrm{m})$ & 2.1 & 2.2 & 1.25 & 1.25 & 0.85 & $1^{3}$ & 1.8 & 1.75 & 2.05 & 2.5 \\
\hline Cell width $^{7}(\mu \mathrm{m})$ & 1.15 & 1.2 & 0.9 & $0.6^{3}$ & 0.45 & $0.4^{3}$ & 0.95 & 0.8 & 0.8 & 1 \\
\hline sMMO & $0^{8}$ & 0 & $1^{9}$ & $0^{10}$ & 1 & $0^{3}$ & 1 & 1 & n.a. ${ }^{11}$ & $1^{12}$ \\
\hline $\mathrm{NaCl}$ tolerance & 3 & 0 & 2.5 & 1 & n.a. ${ }^{11}$ & 2 & n.a. ${ }^{11}$ & 0.8 & 2 & 2 \\
\hline $\mathrm{G}+\mathrm{C} \mathrm{mol} \%$ & 50 & $49.5^{13}$ & $63.6^{9}$ & 62 & n.a. ${ }^{11}$ & $63.4^{14}$ & $62.5^{15}$ & $63^{16}$ & 66 & $66^{12}$ \\
\hline $\begin{array}{l}\text { Forms aggregates, } \\
\text { rosettes or chains }\end{array}$ & 0.25 & 1 & 0 & 0 & 1 & 0 & 1 & 1 & 1 & 1 \\
\hline Grows at $\mathrm{pH} 9$ & 1 & 0 & 0 & 0 & 0 & 0.62 & 1 & 0 & 1 & 1 \\
\hline Grows at $\mathrm{pH} 5$ & 0.25 & 1 & 0 & 0 & n.a. ${ }^{11}$ & 1 & 0 & 1 & 0 & 0 \\
\hline pH optimum & 6.8 & 6.8 & n.a. ${ }^{11}$ & 7 & 7 & 7 & 7 & $5.5^{1}$ & 6.8 & 6.8 \\
\hline $\begin{array}{l}\text { Temperature } \\
\text { optimum }\left({ }^{\circ} \mathrm{C}\right)\end{array}$ & 30 & 23 & 42 & $27^{10}$ & 30 & 28.5 & 27 & 23 & 30 & 28.5 \\
\hline Genome size (bp) & $5,100,000^{8}$ & $4,800,000^{13}$ & $3,300,000^{9}$ & n.a. ${ }^{11}$ & n.a. ${ }^{11}$ & $4,500,000^{14}$ & $4,300,000^{15}$ & $3,900,000^{16}$ & n.a. ${ }^{11}$ & $4,900,000^{12}$ \\
\hline PLFA_PCoA1 & -37.06 & -31.94 & -31.21 & 31.25 & 36.01 & 24.41 & 25.78 & -27.84 & -27.04 & 37.63 \\
\hline PLFA_PCoA2 & -26.48 & -31.30 & -33.47 & -4.09 & -6.78 & 1.53 & 15.41 & 56.78 & 39.83 & -11.43 \\
\hline $\begin{array}{l}\text { Initial growth } \\
\operatorname{rate}^{17}\left(\mathrm{~d}^{-1}\right)\end{array}$ & 0.182 & 0 & 0.042 & 0.503 & 0 & 0.233 & 0.126 & 0.076 & 0.172 & 0.247 \\
\hline
\end{tabular}

Notes: Species abbreviations are as follows: M. lut, Methylobacter luteus; M. tun, Methylobacter tundripaludum; M. cap, Methylococcus capsulatus; M. ech, Methylocystis echinoides; M. hir, Methylocystis hirsuta; M. par, Methylocystis parvus; M. sil, Methylocella silvestris; M. ros, Methylocystis rosea; M. spo, Methylosinus sporium; M. tri, Methylosinus trichosporium.

Sources for all traits of a strain unless otherwise indicated: 1, Bowman et al. (1993); 2, Wartiainen et al. (2006b); 3, Lindner et al. (2007); 4, temperature optimum based on range in Dunfield et al. (2003) and own measurments; 5, Wartiainen et al. (2006a); 6, Whittenbury et al. (1970); 7, middle of length and width distribution reported in literature; 8, Hamilton et al. (2015); 9, Ward et al. (2004), 10, Belova et al. (2013); 11, missing value, mean trait value was used; 12, Stein et al. (2010); 13, Kalyuzhnaya et al. (2015); 14, del Cerro et al. (2012); 15, Chen et al. (2010), 16, https://www.ncbi.nlm.nih.gov/assembly/GCF_000372845.1; 17, based on growth of monocultures under experimental conditions calculated from $\mathrm{CH}_{4}$ oxidation constant $k$, assuming logistic growth.

inocula that we mixed were inversely proportional to the target strain richness, i.e., all microcosms were inoculated with approximately the same total number of cells. We created 10 different strain compositions at each diversity level except for the 10 -strain mixture. The compositions were created by randomly selecting strains, with the restriction that each strain was present in an equal number of mixtures at each diversity level. This ensured that strain richness was varied independently of the average composition found at each diversity level. Each specific composition was replicated in seven separate microcosms, which were placed together in an incubation jar. The most diverse mixture with 10 strains was replicated an additional four times, in four separate jars. One microcosm per jar was immediately frozen at $-80^{\circ} \mathrm{C}$ to assess the strain composition at the beginning of the experiment. The microcosms were then closed with gas-permeable cotton stoppers to minimize the risk of cross-contamination. The jars containing the microcosms were placed in incubators that ran a $24-\mathrm{h}$ temperature cycle (with four periods of $6 \mathrm{~h}$ each: $24^{\circ} \mathrm{C}, 26^{\circ} \mathrm{C}$, $28^{\circ} \mathrm{C}, 26^{\circ} \mathrm{C}$ ). Every two days, the jars were ventilated to remove the $\mathrm{CO}_{2}$ produced and new $\mathrm{CH}_{4}$ was added. The average starting concentration of $\mathrm{CH}_{4}$ was $9,120 \pm 1,140 \mu \mathrm{L}$ $\mathrm{CH}_{4} / \mathrm{L}$ (mean $\pm \mathrm{SD}$ ). One microcosm each was removed from each jar and frozen after 4, 8, 16, 24, 32, and $44 \mathrm{~d}$ of incubation for later analysis (see Appendix S1: Fig. S2 for an overview of the experimental setup and sampling).

\section{$\mathrm{CH}_{4}$ consumption}

In order to quantify $\mathrm{CH}_{4}$ consumption, $30 \mathrm{~mL}$ samples were taken from the headspace of each jar at the beginning and at the end of each two-day incubation period, for a total of $32 \mathrm{~d}$. The $\mathrm{CH}_{4}$ concentration of these samples was determined by gas chromatography (Agilent $7890 \mathrm{~N}$ gas chromatograph; $\mathrm{CH}_{4}$ was detected with a flame ionization detector; $12^{\prime}$ Porapak Q column; isothermic at $80^{\circ} \mathrm{C}$; He carrier gas; $\mathrm{CO}_{2}$ was determined on the same detector after reducing $\mathrm{CO}_{2}$ with $\mathrm{H}_{2}$ on a Ni-catalyst; Agilent Technologies Inc., Santa Clara, California, USA). $\mathrm{CH}_{4}$ consumption was then determined as apparent first-order oxidation rate constant $\left(d c / d t=-c \times k\right.$, where $c$ is the headspace $\mathrm{CH}_{4}$ concentration and $k$ is the rate constant) by estimating $k$ through linear regression of $\ln (c)$ against time $(t)$. We standardized $k$ values by dividing these by the number of microcosms in the jar.

\section{DNA extraction}

We extracted DNA from the replicate microcosms frozen after 0,16 , and $44 \mathrm{~d}$ of incubation. DNA was extracted from the entire soil in the microcosms using the xhantogenatebased extraction method described in Tillett and Neilan (2000) with some adjustments (Appendix S1: Table S1). 
Extracted DNA was purified using the PowerClean Pro DNA Clean-Up Kit (MoBio, Carlsbad, California, USA) according to the manufacturer's instruction.

\section{Sequencing of pmoA and $16 S$ gene fragments}

To assess changes in community composition, we amplified the variable regions V1 and V2 of the 16S rRNA gene from total nucleic acids extracted after 0,16 , and $44 \mathrm{~d}$ of incubations (using primers $9 \mathrm{bF}$ and $341 \mathrm{R}$; see Appendix S1: Tables S2 and S3 for details). The fragments of the gene for subunit A of the particulate methane monooxygenase (pmoA) were sequenced for the samples taken after $16 \mathrm{~d}$ only (primers A189F and M661R; see Appendix S1: Tables S2 and S3 for details). The PCR products obtained were purified with the GeneJET PCR purification kit (Thermo Scientific, Waltham, Massachusetts, USA) and quantified with the 2100 Bioanalyzer high sensitivity DNA kit (Agilent Technologies, Santa Clara, California, USA). Barcoding using the Fluidigm access array technology and paired-end sequencing of the amplified region with the Illumina MiSeq v3 platform was carried out at the Genome Quebec Innovation Center, Montreal, Canada.

Analysis of the $16 \mathrm{~S}$ rRNA and the pmo $A$ gene sequences was performed according to a customized pipeline published in Frey et al. (2016). However, as we knew the strains present, we did not perform OTU clustering and taxonomic assignment but instead mapped the reads directly against the known 16S rRNA and pmo $A$ sequences of our strains. We used algorithms from USEARCH v8 (Edgar 2010) unless otherwise stated. Forward and reverse read pairs were merged using the fastq_mergepairs algorithm (Edgar and Flyvbjerg 2015, minimum overlap between forward and reverse read pairs 50 base pairs [bp], minimum merged read length of $300 \mathrm{bp}$ ). PCR primers were detected and removed with Cutadapt (Martin 2011). Trimmed sequences were then quality-filtered using the fastq_filter function (Edgar and Flyvbjerg 2015) with a maximum expected error threshold of one. All quality-filtered reads were mapped against the known 16S rRNA and pmoA sequences of the methanotroph strains we had used to create the experimental biodiversity gradient (sequences were retrieved from GenBank but verified by Sanger-sequencing the pure methanotroph cultures) using the usearch_global algorithm (Edgar 2010; options $\mathrm{id}=99$, maxrejects $=0$, maxaccepts $=0$, and top hit only). We then counted the number of matched sequences of each strain in each sample.

Presense/absence of strains based on pmoA data (day 16) matched the ones for 16S. Since Methylocella silvestris was not detectable in the pmoA sequence data due to a lack of pmo $A$ gene we used $16 \mathrm{~S}$ rRNA-based abundance data to calculate diversity indices. We calculated the relative abundance of each strain in all the samples based on the number of matching 16S rRNA gene copies. From this, the realized strain richness was calculated (i.e., number of strains that remained after a certain time) by counting the strains with a relative abundance of more than $0.1 \%$; this minimum abundance was chosen to exclude effects of spurious contaminations. We further calculated the Shannon diversity index $\left(H^{\prime}\right)$ and from that effective strain richness $\left(e^{H^{\prime}}\right)$, which gives the low-abundant strains a lower weight. Right after inoculation (day 0), not enough DNA could be extracted to obtain a reliable diversity estimate by sequencing. Diversity indices for day 16 were similar to the ones for day 44, and we therefore based our calculations of realized and effective strain richness on $16 \mathrm{~S}$ rRNA gene sequencing information from day 44 .

\section{Numbers of pmoA copies}

We determined the abundances of methanotrophic pmo $A$ gene copies by quantitative PCR (StepOne real-time PCR system Applied Biosystems, Foster City, California, USA; see Appendix S1: Tables S2 and S3 for details). The number of $p m o A$ gene copies in the genome were not known for all strains in our experiment; however, where known, methanotrophs had one or two gene copies except for Methylocella silvestris, which has a soluble instead of a particulate methane mono-oxygenase and therefore lack the pmo $A$ gene. Nevertheless, we used number of pmo $A$ copies as proxy of methanotroph community size; $16 \mathrm{~S}$ sequence analysis indicated that Methylocella silvestris was among the least abundant methanotrophs, and the lack of pmo $A$ gene in this strain likely did not substantially bias our community size estimate. A serial dilution of purified DNA from Methylococcus capsulatus (quantified with the Qubit fluorometer; Invitrogen, Carlsbad, California, USA) was included in duplicates in each run to determine the calibration curve. The efficiency values of the runs calculated from the slopes of the calibration curves were between $72 \%$ and $83 \%$.

\section{Functional and phylogenetic diversity}

To estimate phylogenetic diversity, we calculated a phylogenetic tree (UPGMA) with the EMBL-EBI MUSCLE online tool (Edgar 2004, McWilliam et al. 2013) using pmo A sequences of our strains plus a collection of 67 additional sequences (retrieved from GenBank using accessions from Lüke and Frenzel 2011). To include Methylocella silvestris in the tree, which doesn't have a pmo $A$ gene, we used the pmo $A$ sequence of Methylocapsa aurea instead (accession no FN433470). Of the available methanotrophs that have a pmo $A$ gene, $M$. aurea shows the highest $16 \mathrm{~S}$ rRNA sequence similarity to $M$. silvestris. The phylogenetic diversity (PD) of each community was calculated as the total branch length of the subtree defined by the strains in the mixture (calcPD function from library pdiv, available online). ${ }^{8}$ Functional diversity (FD) was calculated similarly, using a trait dendrogram (Petchey and Gaston 2006) constructed from traits available from the literature (Table 1; calcFD function in $\mathrm{R}$ library pdiv). We used Gower distances in the distance matrix underlying the trait dendrogram because this metric is preferable when combining continuous and binary traits.

\section{Statistical analysis}

We used analysis of variance based on general linear models (R 3.0; R Core Team 2013) to test for relationships between each of our measures of diversity (applied, realized and effective strain richness, PD, FD) and the methanotroph activity and community size. As is standard in the analysis

\footnotetext{
${ }^{8}$ https://github.com/pascal-niklaus/pdiv
} 
of biodiversity experiments (Schmid et al. 2017), we used the specific composition of the community as replicate; this was achieved by averaging the four technical replicates of the 10-strain community before analysis. All measures of diversity were included in the model in log-transformed form; the reason for this is that typically BEF relationships are "positive decelerating" (Cardinale et al. 2011), and that this relationship is linearized by log-transforming diversity.

The main dependent variables were the cumulative $\mathrm{CH}_{4}$ consumption over the duration of the incubation (indicated by the mean first order $\mathrm{CH}_{4}$ consumption rate constant), and the net methanotrophic growth (indicated by pmo $A$ copy numbers at day 44). To achieve a homoscedastic distribution of the residuals, we square root-transformed the first-order rate constant for $\mathrm{CH}_{4}$ consumption. To test whether the presence of particular strains in the communities had an important effect on our dependent variables beyond the overall effect of strain diversity, we also added presence/absence contrasts for the 10 strains (effects of the different strains tested in separate models).

Inspection of data revealed three distinct phases in the course of the incubation. In addition to analyzing cumulated $\mathrm{CH}_{4}$ consumption, we therefore also analyzed mean $\mathrm{CH}_{4}$ consumption for the initial phase of the study where fast growth was observed (days 0 to 10 ), the middle period with highest $\mathrm{CH}_{4}$ consumption (days 12 to 20), and the final phase of the incubation that was characterized by lower $\mathrm{CH}_{4}$ uptake rates (days 22 to 32 ).

We further inspected whether effects of strain richness on $\mathrm{CH}_{4}$ consumption were related to methanotrophic community size. To do so, we assigned the pmo $A$ copy numbers of day 0,16 , and 44 to the initial, middle, and final phase, respectively. Then, for each time period, we fitted linear models in which applied strain richness was followed by pmo $A$ copy number, and vice versa. The first model yielded tests for overall effects of strain richness, whereas the second model yielded tests for effects of strain richness beyond any effect that was already explained by variation in community size.

The different strains exhibited different temporal dynamics in monoculture, with some performing particularly well at the beginning of the experiment and some at the end. We therefore tested for effects of temporal complementarity on $\mathrm{CH}_{4}$ consumption and community size by estimating the size of the "community niche" CN (Salles et al. 2009): for each measurement day, we determined the maximum $\mathrm{CH}_{4}$ consumption rate of the monocultures of the strains present in the mixture, and summed these values. $\mathrm{CN}$ thus quantifies the cumulative $\mathrm{CH}_{4}$ consumption that would be achieved if community-level consumption was determined by the strain performing best on that particular day. We used $\mathrm{CN}$ as explanatory variable in further linear models, fitting this term before and after strain richness.

We quantified the over-performance (net biodiversity effect) of all mixtures for both $\mathrm{CH}_{4}$ consumption and pmo $\mathrm{A}$ copy numbers. For $\mathrm{CH}_{4}$ consumption, over-performance was calculated from mean consumption rates over the entire experiment. For pmo $A$ copy numbers, we calculated overperformance (overyielding) for day 44 . The expected value for each mixture was calculated by averaging the values measured for the monocultures microcosms in the experiment of the strains present in the mixture. The over-performance was calculated as observed minus expected $\mathrm{CH}_{4}$ consumption and pmo $A$ copy numbers. We used a one-sample, onesided Student's $t$ test to test for over-performance of mixtures in relation to monocultures. We then used linear models to test whether the over-performance of mixtures depended on the number of strains they contained. Finally, we tested whether over-yielding of pmo A copies correlated with the over-performance of the same mixtures with respect to $\mathrm{CH}_{4}$ consumption.

\section{RESUlTS}

\section{Community compositions}

We obtained $(41 \pm 11) \times 10^{3}$ bacterial $16 \mathrm{~S}$ rRNA sequences per microcosm (in total $5.4 \times 10^{6} 16 \mathrm{~S}$ rRNA sequences $[$ mean $\pm \mathrm{SE}])$ and $(41 \pm 8.4) \times 10^{3}$ pmo $A$ sequences per microcosm (in total $1.8 \times 10^{6}$ pmo $A$ sequences). After quality control, $1.3 \times 10^{6} 16 \mathrm{~S}$ rRNA sequences could be mapped to 1 of the 10 strains used for creating the communities and $2.8 \times 10^{5}$ pmo $A$ sequences could be mapped to the nine strains that possess a pmo $A$ gene. Overall, the number of $16 \mathrm{~S}$ rRNA sequences that belonged to 1 of out 10 strains increased over the course of the experiment, while the number of other non-methanotroph 16S rRNA sequences decreased. The 16S rRNA sequences that did not match any of the 10 strains we added was likely DNA from various soil bacteria that had remained in the soil matrix. This DNA slowly degraded over the course of the experiment.

The starting composition of the methanotroph communities based on the 16S rRNA sequences was consistent with the strains that were added. While the number of sequences varied between samples, within sample the number of sequences for each strain was quite even. The composition of methanotroph communities in the different mixtures changed over time. In general, the strain distribution became more uneven through time, with experimental communities dominated by one or a few strains at the end of the experiment. The different strains performed differently well in our microcosms: Methylocystis echinoides became dominant in many but not all mixtures; in some communities other strains became dominant even when Methylocystis echinoides was present. No single strain was entirely lost from the experiment but Methyloccocus capsulatus and Methylobacter tundripaludum disappeared from $87 \%$ and $50 \%$ of the communities in which they were originally present, respectively. Methylocystis echinoides and Methylosinus trichosporium persisted in all communities. The extinction of the other strains ranged from $12 \%$ to $37 \%$ of the communities.

Despite these strain-specific effects, the experimental methanotroph diversity gradient persisted throughout the study. Both realized strain richness and Shannon-based effective strain richness (calculated based on 16S rRNA sequences after $44 \mathrm{~d}$ ) were highly significantly correlated with applied strain richness (Pearson's product moment correlation $r=0.82$ and $0.46, P<0.001$ for both indices; Appendix S1: Fig. S1). The realized strain richness was higher than expected in some monocultures and two-strain mixtures, likely because of cross-contamination during handling in the laboratory. 


\section{Community size}

Average pmoA gene copy numbers increased throughout the experiment, with the largest increase between days 0 and 16. After $44 \mathrm{~d}$, pmo $A$ copy numbers were significantly positively correlated with applied strain richness (applied strain richness, $P=0.03$; Fig. 1) and marginally significantly with realized strain richness (realized strain richness, $P=0.06$ ). These effects already began to develop earlier (day 16) but were not statistically significant. Effective strain richness, phylogenetic diversity (PD), and functional diversity (FD) were not statistically significantly related to pmo $A$ copy numbers at any date.

When including the presence/absence of the strains in the model, communities with Methylocystis parvus had significantly higher copy numbers $(P<0.01)$ and Methylosinus trichosporium $(P<0.05)$ significant lower copy numbers (test after adjusting for strain richness).

\section{$\mathrm{CH}_{4}$ consumption}

Over the course of the experiment, $\mathrm{CH}_{4}$ consumption increased until day 10, remained stable until day 16 and then decreased (Fig. 2). Differences in $\mathrm{CH}_{4}$ consumption between the samples and over time ranged from almost no consumption to consuming nearly all the added $\mathrm{CH}_{4}$ within the twoday period. Over the entire measurement period of $32 \mathrm{~d}$, average $\mathrm{CH}_{4}$ consumption increased statistically significantly with applied strain richness $(P=0.03$; Fig. 3$)$. Neither realized nor effective strain richness, FD or PD explained any significant fraction of the variation observed in $\mathrm{CH}_{4}$ consumption. Models including presence-absence contrasts after the effect of applied strain richness indicated that communities with either Methylobacter tundripaludum $(P<0.01)$ or Methylocella silvestris $(P<0.05)$ consumed particularly little $\mathrm{CH}_{4}$.

When analyzing the period of most rapid growth separately (days $0-10), \mathrm{CH}_{4}$ consumption increased significantly with applied strain richness $(P<0.05)$, but not with realized

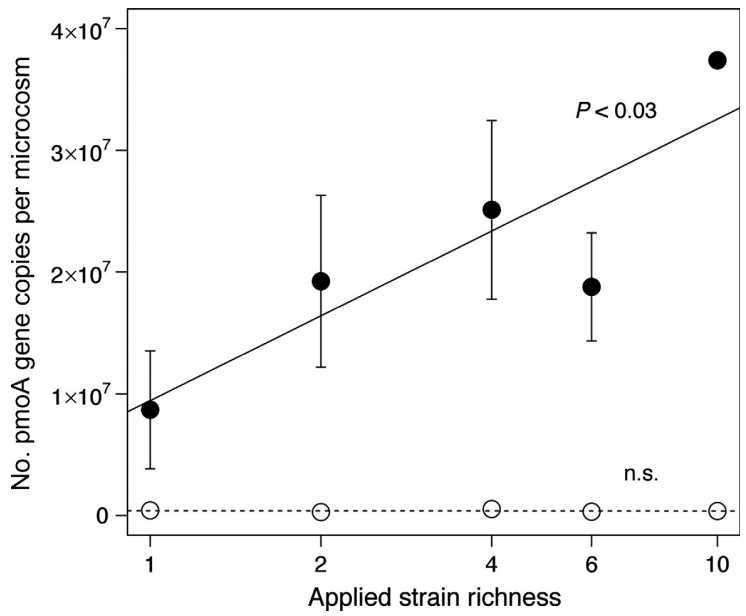

FIG. 1. Mean number of $p m o A$ gene copies per microcosm by applied strain richness at the beginning (open circle, dashed line, not significant [n.s.]) and at the end of the experiment (solid circle, solid line, $P<0.03$ ). Error bars show the standard error for the different compositions ( $n=10$ except for the 10-strain mixture, which consists of only one specific composition that was replicated four times). strain richness, effective strain richness, PD, or FD. For the intermediate period characterized by high $\mathrm{CH}_{4}$ consumption (days 12-20), there was no significant relationship between $\mathrm{CH}_{4}$ consumption and any of the diversity measures though effects of applied strain richness were marginally significant $(P=0.06)$. We did not find any diversity effects for the last phase of the experiment (days 22-32).

When including numbers of pmo $A$ gene copies as an explanatory covariate before applied strain richness, in the first and intermediate period, effects of applied strain richness were statistically significant $(P<0.05)$ but $p m o A$ copy numbers were not. In the final period, effects of strain richness were no longer significant but effects of pmo $A$ copy number were $(P<0.05)$.

The community niche metric $(\mathrm{CN})$ we calculated from temporal monoculture $\mathrm{CH}_{4}$ consumption profiles (Appendix S1: Fig. S3) was highly correlated with applied strain richness (Pearson's product moment correlation $r=0.86 ; P<0.001$ ) but $\mathrm{CN}$ did not explain significant amounts of variation in cumulated $\mathrm{CH}_{4}$ consumption, regardless of whether $\mathrm{CN}$ was fitted before or after applied species richness.

\section{Over-performance of strain mixtures and effects of individual strains}

We observed an over-performance of strain mixtures both with respect to $\mathrm{CH}_{4}$ consumption (first-order uptake rate constant) and community size (number of pmo $A$ gene copies). Almost all strain mixtures consumed more $\mathrm{CH}_{4}$ than would have been expected based on the mean $\mathrm{CH}_{4}$ consumption of their monocultures $(P<0.001$; Fig. 4$)$. The net biodiversity effect (observed minus expected $\mathrm{CH}_{4}$ consumption) was positive but not significantly related to any of the diversity indices.

Mixtures also showed over-yielding with respect to number of pmo $A$ gene copies. As expected based on the constant amount of inoculum added, there was no statistically significant difference between observed and expected number of copies at the start of the incubation. After $44 \mathrm{~d}$ of growth, the numbers of $p m o A$ gene copies of mixtures exceeded average numbers of copies of monocultures of the component strains $(P<0.001)$. Similar to $\mathrm{CH}_{4}$ consumption rates, the net biodiversity effect on number of $p m o A$ gene copies was positive but not significantly related to any of the diversity indices.

Net biodiversity effects for $\mathrm{CH}_{4}$ consumption and for community size were positively correlated (Pearson's product moment correlation $r=0.45 ; P<0.004 ; R^{2}=0.18$ ). Strain mixtures that exhibited higher than expected $\mathrm{CH}_{4}$ consumption based on their component monocultures also produced larger communities than expected.

\section{DisCUSSION}

Here we explored the biodiversity-ecosystem-function (BEF) relationship in a specialized group of bacteria, the methanotrophs. We demonstrated that increased methanotrophic strain diversity significantly promotes $\mathrm{CH}_{4}$ consumption rates, growth, and resulting community size. In other words, mixtures of strains consumed $\mathrm{CH}_{4}$, on average, at higher rates and grew more than monocultures of the strains they contain. 


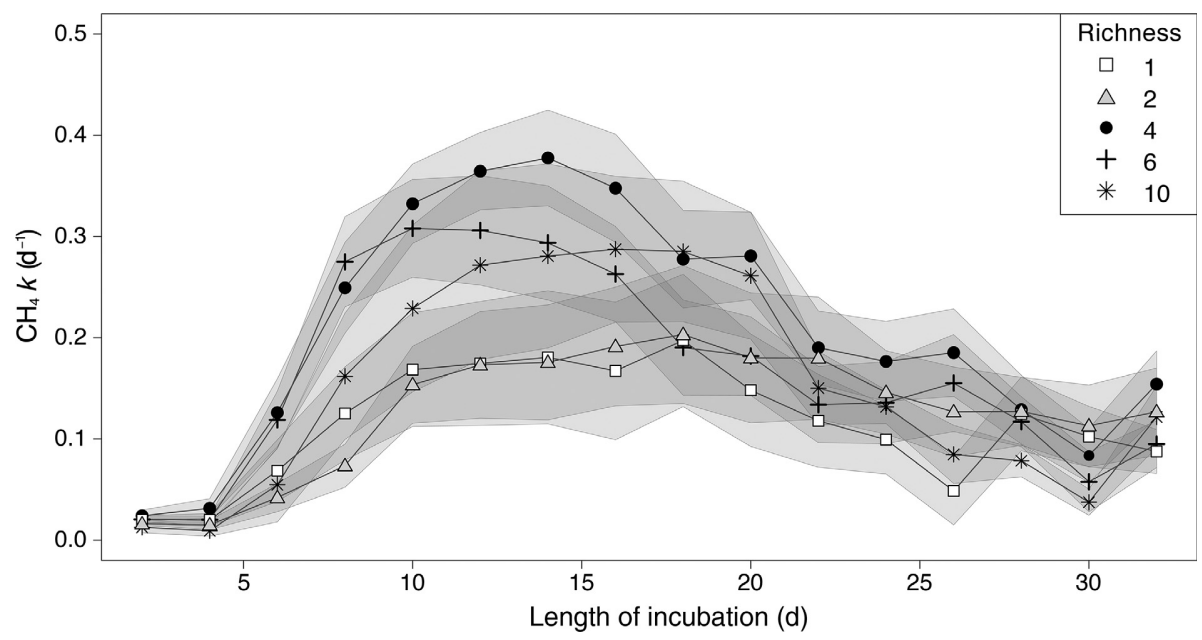

FIG. 2. Temporal change in apparent first-order $\mathrm{CH}_{4}$ uptake rate constants $k$ for each diversity level. Lines show mean values with gray areas indicating standard errors using different communities as replicates $(n=10)$ except for the 10-strain mixture where technical replicates $(n=4)$ of the same composition are used.

While positive BEF relationships have been found as a general trend in studies of plant communities, this study is one of a few experimental studies available to date that demonstrate a positive BEF relationship in communities of microorganisms (e.g., Wohl et al. 2004, Bell et al. 2005, Replansky and Bell 2009, Salles et al. 2009, 2012, Philippot et al. 2013, Delgado-Baquerizo et al. 2016). In other studies no significant BEF relationship was found (e.g., Griffiths et al. 2000, 2001, Seghers et al. 2003, Wertz et al. 2006, 2007), and some microbial model systems even revealed negative BEF relationships, which were ascribed to more frequent antagonistic interactions in more diverse system, for example through the production of toxins (Jousset et al. 2011, Becker et al. 2012). Interestingly, in our study, methanotrophic strain richness was the best predictor of effects on $\mathrm{CH}_{4}$ consumption and methanotrophic growth. Effective strain richness did not explain much variation in our data, indicating that strains low in abundance are important for

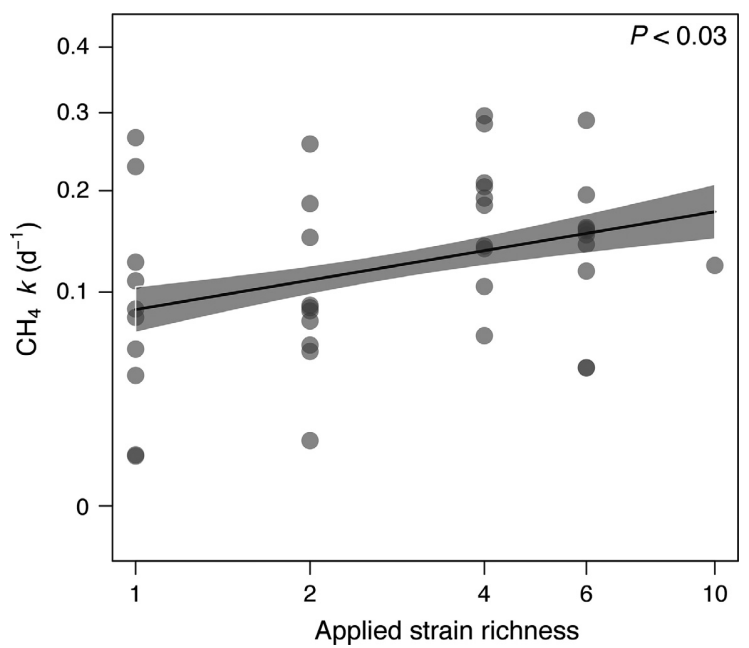

FIG. 3. The mean first-order $\mathrm{CH}_{4}$ uptake rate constant $k$ (averages over the $32 \mathrm{~d}$ of measurement) increased significantly with the logarithm of applied strain richness $(P<0.03)$. Line and error bars show model-predicted means and standard errors. Note square-root transformed scale of the $y$-axis. the maintenance of ecosystem functioning (Bodrossy et al. 2003, Bodelier et al. 2013). Ultimately, BEF relationships arise from functional differences among strains; functional diversity (FD) therefore often explains more variation in community functioning than strain richness alone (Krause et al. 2014b), but we found no relationship between FD and $\mathrm{CH}_{4}$ oxidation rates or growth of methanotrophs in our study. In particular, FD did not explain significant fractions of variation in our data that were not already explained by strain richness. One reason for the lack of explanatory power of our FD metric may have been that we did not include the trait measures or combinations of these that were relevant in our experimental context. Or alternatively, that we included

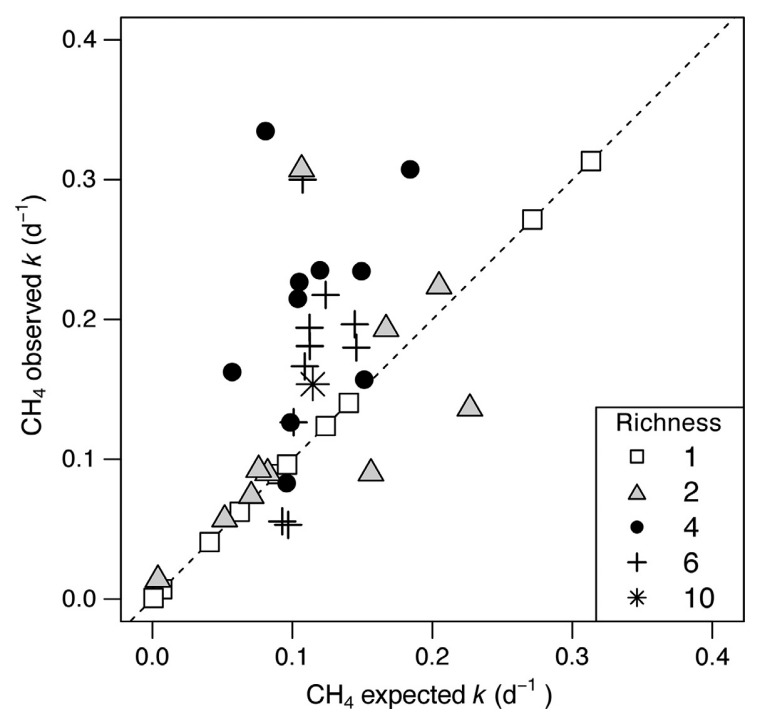

FIG. 4. Over-performance of strain mixtures relative to monocultures with respect to $\mathrm{CH}_{4}$ consumption (measured as apparent first-order $\mathrm{CH}_{4}$ consumption constant). For most mixtures, the observed $\mathrm{CH}_{4}$ oxidation constant $k$ is higher than the expected $\mathrm{CH}_{4}$ oxidation constant $k$. The expected constant $k$ was calculated by taking the mean of the values measured for the monocultures of the strains that were in the mixture. The dashed line indicates the 1:1 relationship. 
traits that have no functional implications and therefore added "noise" to our predictor. Growth-relevant traits are more difficult to determine for microorganisms than for higher plants, and only a few traits were consistently available for all strains. In plants, traits such as specific leaf area or root-shoot ratios are known to define growth strategies. In analogy, we focused on maximum growth rate and measures that may have allometric implications such as the size of cells or whether these form aggregates. We reasoned that these could affect surface to volume ratios and therefore specific activities. Other obvious candidate traits are the kinetic characteristics of $\mathrm{CH}_{4}$ consumption, but these are notoriously difficult to estimate because $\mathrm{CH}_{4}$ uptake rates in laboratory systems are affected by diffusion limitations in the medium in which the organism grows (Dunfield and Conrad 2000). Also, these traits may be very plastic, allowing acclimation to environments with different resource supply. Finally, all methanotrophs critically rely on $\mathrm{CH}_{4}$ supply, which may lead to phenotypic convergence, in particular in the absence of spatiotemporal heterogeneity in $\mathrm{CH}_{4}$ concentrations. Niche complementarity that promotes biodiversity effects may thus be in other functional dimensions, which could for example be related to biotic interactions or to the uptake of other nutrients.

Some of the difficulties in determining the relevant functional traits can be circumvented by using phylogenetic distance as proxy of niche complementarity. Several studies have shown that this can work well in communities of higher plants, suggesting that the relevant traits are conserved as species evolve (Flynn et al. 2011). However, this may not be true for all organisms and traits (Crisp and Cook 2012). Evolutionary change can happen rapidly in microbes, potentially leading to a weaker phylogenetic signal for less conserved traits (Martiny et al. 2013). In our study, the phylogenetic diversity (PD) of methanotrophs did not predict their community-level functioning. Methanotrophs in particular have shown a weak phylogenetic signal in physiological traits relevant to $\mathrm{CH}_{4}$ oxidation (Krause et al. 2014a), suggesting that the traits are not highly conserved. Additionally, some of the strains used in this experiment are rather closely related, which might underestimate the functional diversity that is there and which promotes community-level functioning. For example, differences in metabolic responses have been found even between closely related strains of methanotrophs (Hoefman et al. 2014). So PD may not be a good proxy for FD in the case of methanotrophs and not suitable for explaining BEF relationships.

In our experiment, effects of diversity first manifested in higher $\mathrm{CH}_{4}$ consumption and towards the end of the experiment in a larger community size. This sequence of events can be understood in the light of $\mathrm{CH}_{4}$ assimilation being the process that drives the growth of the communities. Eller and Frenzel (2001) reported a similar pattern in microcosms with rice paddy model systems in which activity and methanotroph cell counts were negatively correlated through time and cell counts increased even after activity had started to decrease.

Both $\mathrm{CH}_{4}$ consumption and methanotrophic community growth show over-yielding in mixtures compared to monocultures, i.e., on average, mixtures consumed more $\mathrm{CH}_{4}$ and grew more than would have been expected based on the performance of monocultures of the strains they contained.
Over-yielding of community size and $\mathrm{CH}_{4}$ consumption were positively correlated, indicating that mixtures that consumed $\mathrm{CH}_{4}$ at particularly high rates also grew more. Biodiversity effects with macro-organisms are often classified as complementarity or selection effects by using statistical partitioning schemes (Loreau and Hector 2001). In our study, almost all mixtures showed over-yielding, suggesting that biodiversity effects were not just driven by a few very productive strains (which would be typical for sampling-type effects). Our data did not reveal evidence for large effects of the presence of particular strains. This observation is in agreement with the assumption that complementarity effects drove the observed diversity effects. However, we were not able to test these in a more strict sense because we could not trace back community-level measurements to contributions of particular strains, which is required for, e.g., the additive partitioning method (Loreau and Hector 2001). Monoculture data revealed different growth rates and different periods of maximum activity for the different strains, suggesting that temporal complementarity could have been important. However, temporal complementarity, quantified as community niche, did not explain much variation in our data. Nevertheless, such a temporal niche partitioning may help to stabilize methanotrophic activity in natural and semi-natural wetlands. Environmental changes due to seasonality, management (e.g., water level changes, fertilizer application, crop harvesting), or irregular climatic events (e.g., drought) will disturb methanotrophic communities. These will respond with a temporal succession of species (Collet et al. 2015, Ho et al. 2016a, b), a process that will only stabilize function through time if a sufficiently diverse pool of taxa is available.

In this study, we show a significant biodiversity-ecosystemfunction relationship in methanotrophic bacteria. To date, only a few other studies are available in which the diversity of microorganisms was manipulated directly via assembly of pure strains (Wohl et al. 2004, Bell et al. 2005, Salles et al. 2009, 2012, Gravel et al. 2011), allowing to infer causal effects of biodiversity on functioning (Krause et al. 2014b). Our study demonstrates that BEF relationships can show similar patterns in microbial communities to those found in communities of higher organisms, for example plants. Our findings support the importance of biological diversity for driving particular ecosystem functions. Moreover, they also demonstrate that BEF relationships show similar patterns at different scales and in different types of organisms and are therefore likely widespread in nature. Methanotrophs are important for the regulation of $\mathrm{CH}_{4}$ emissions (Le Mer and Roger 2001), as they act as a "filter" and oxidize the $\mathrm{CH}_{4}$ produced in anaerobic environments before it enters the atmosphere. We show in our experiment that a loss in diversity significantly decreases the $\mathrm{CH}_{4}$ oxidation and observational studies suggest that diversity may also important in natural methanotrophic communities for maintaining $\mathrm{CH}_{4}$ oxidation and to increase its temporal stability (Mohanty et al. 2006, Levine et al. 2011, Siljanen et al. 2011, Bodelier et al. 2013).

\section{ACKNOWLEDGMENTS}

This project was funded by the Swiss National Science Foundation (grant 144065 to P. A. Niklaus) and the University of Zurich. We gratefully acknowledge Sascha Krause and Marion Meima for the provision of some of the methanotroph cultures we used. We thank 
René Husi and Andy Lutz for help with laboratory analysis. We further are indebted to Kentaro Shimizu and the Genetic Diversity Centre (GDC), ETH Zurich for generous help with laboratory facilities. P. A. Niklaus acknowledges support from the University of Zurich Research Priority Programme Global Change and Biodiversity.

\section{Literature Cited}

Aarssen, L. W. 1997. High productivity in grassland ecosystems: Effected by species diversity or productive species? Oikos 80:183-184.

Balvanera, P., A. B. Pfisterer, N. Buchmann, J. S. He, T. Nakashizuka, D. Raffaelli, and B. Schmid. 2006. Quantifying the evidence for biodiversity effects on ecosystem functioning and services. Ecology Letters 9:1146-1156.

Becker, J., N. Eisenhauer, S. Scheu, and A. Jousset. 2012. Increasing antagonistic interactions cause bacterial communities to collapse at high diversity. Ecology Letters 15:468-474.

Bell, T., J. A. Newman, B. W. Silverman, S. L. Turner, and A. K. Lilley. 2005. The contribution of species richness and composition to bacterial services. Nature 436:1157-1160.

Belova, S. E., I. S. Kulichevskaya, P. L. E. Bodelier, and S. N. Dedysh. 2013. Methylocystis bryophila sp. nov., a facultatively methanotrophic bacterium from acidic Sphagnum peat, and emended description of the genus Methylocystis (ex Whittenbury et al. 1970) Bowman et al. 1993. International Journal of Systematic and Evolutionary Microbiology 63:1096-1104.

Bier, R. L., et al. 2015. Linking microbial community structure and microbial processes: An empirical and conceptual overview. FEMS Microbiology Ecology 91:fiv113.

Bodelier, P. L. E., and A. K. Steenbergh. 2014. Interactions between methane and the nitrogen cycle in light of climate change. Current Opinion in Environmental Sustainability 9-10:26-36.

Bodelier, P. L. E., M. Meima-Franke, C. A. Hordijk, A. K. Steenbergh, M. M. Hefting, L. Bodrossy, M. von Bergen, and J. Seifert. 2013. Microbial minorities modulate methane consumption through niche partitioning. ISME Journal 7:2214-2228.

Bodrossy, L., N. Stralis-Pavese, J. C. Murrell, S. Radajewski, A. Weilharter, and A. Sessitsch. 2003. Where less may be more: How the rare biosphere pulls ecosystems strings. Environmental Microbiology 5:566-582.

Bowman, J. P., L. I. Sly, P. D. Nichols, and A. C. Hayward. 1993. Revised taxonomy of the methanotrophs: Description of Methylobacter gen. nov., emendation of Methylococcus, validation of Methylosinus and Methylocystis species, and a proposal that the Family Methylococcaceae includes o. International Journal of Systematic Bacteriology 43:735-753.

Cardinale, B. J., K. L. Matulich, D. U. Hooper, J. E. Byrnes, E. Duffy, L. Gamfeldt, P. Balvanera, M. I. O'Connor, and A. Gonzalez. 2011. The functional role of producer diversity in ecosystems. American Journal of Botany 98:572-592.

Cardinale, B. J., et al. 2012. Biodiversity loss and its impact on humanity. Nature 486:59-67.

Chen, Y., A. Crombie, M. T. Rahman, S. N. Dedysh, W. Liesack, M. B. Stott, M. Alam, A. R. Theisen, J. C. Murrell, and P. F. Dunfield. 2010. Complete genome sequence of the aerobic facultative methanotroph Methylocella silvestris BL2. Journal of Bacteriology 192:3840-3841.

Collet, S., A. Reim, A. Ho, and P. Frenzel. 2015. Recovery of paddy soil methanotrophs from long term drought. Soil Biology and Biochemistry 88:69-72.

Crisp, M. D., and L. G. Cook. 2012. Phylogenetic niche conservatism: What are the underlying evolutionary and ecological causes? New Phytologist 196:681-694.

Dedysh, S. N., N. S. Panikov, and J. M. Tiedje. 1998. Acidophilic methanotrophic communities from Sphagnum peat bogs. Applied and Environmental Microbiology 64:922-929.

del Cerro, C., J. M. García, A. Rojas, M. Tortajada, D. Ramón, B. Galán, M. A. Prieto, and J. L. García. 2012. Genome sequence of the methanotrophic poly- $\beta$-hydroxybutyrate producer Methylocystis parvus OBBP. Journal of Bacteriology 194:5709-5710.
Delgado-Baquerizo, M., L. Giaramida, P. B. Reich, A. N. Khachane, K. Hamonts, C. Edwards, L. A. Lawton, and B. K. Singh. 2016. Lack of functional redundancy in the relationship between microbial diversity and ecosystem functioning. Journal of Ecology 104:936-946.

Dunfield, P. F., and R. Conrad. 2000. Starvation alters the apparent half-saturation constant for methane in the type II methanotroph Methylocystis strain LR1. Applied and Environmental Microbiology 66:4136-4138.

Dunfield, P. F., W. Liesack, T. Henckel, R. Conrad, and R. Knowles. 1999. High-affinity methane oxidation by a soil enrichment culture containing a type II methanotroph. Applied and Environmental Microbiology 65:1009-1014.

Dunfield, P. F., V. N. Khmelenina, N. E. Suzina, Y. A. Trotsenko, and S. N. Dedysh. 2003. Methylocella silvestris sp. nov., a novel methonotroph isolated from an acidic forest cambisol. International Journal of Systematic and Evolutionary Microbiology 53:1231-1239.

Edgar, R. C. 2004. MUSCLE: Multiple sequence alignment with high accuracy and high throughput. Nucleic Acids Research 32: 1792-1797.

Edgar, R. C. 2010. Search and clustering orders of magnitude faster than BLAST. Bioinformatics 26:2460-2461.

Edgar, R. C., and H. Flyvbjerg. 2015. Error filtering, pair assembly and error correction for next-generation sequencing reads. Bioinformatics 31:3476-3482.

Eller, G., and P. Frenzel. 2001. Changes in activity and community structure of methane-oxidizing bacteria over the growth period of rice. Applied and Environmental Microbiology 67:2395-2403.

Fetzer, I., K. Johst, R. Schäwe, T. Banitz, H. Harms, and A. Chatzinotas. 2015. The extent of functional redundancy changes as species' roles shift in different environments. Proceedings of the National Academy of Sciences USA 112:14888-14893.

Flynn, D. F. B., N. Mirotchnick, M. Jain, M. I. Palmer, and S. Naeem. 2011. Functional and phylogenetic diversity as predictors of biodiversity-ecosystem-function relationships. Ecology 92: 1573-1581.

Frey, B., T. Rime, M. Phillips, B. Stierli, I. Hajdas, F. Widmer, and M. Hartmann. 2016. Microbial diversity in European alpine permafrost and active layers. FEMS Microbiology Ecology 92:1-17.

Gravel, D., T. Bell, C. Barbera, T. Bouvier, T. Pommier, P. Venail, and N. Mouquet. 2011. Experimental niche evolution alters the strength of the diversity-productivity relationship. Nature 469:89-92.

Griffiths, B. S., et al. 2000. Ecosystem response of pasture soil communities to fumigation-induced microbial diversity reductions: an examination of the biodiversity-ecosystem function relationship. Oikos 90:279-294.

Griffiths, B., K. Ritz, R. Wheatley, H. Kuan, B. Boag, S. Christensen, F. Ekelund, S. Sørensen, S. Muller, and J. Bloem. 2001. An examination of the biodiversity-ecosystem function relationship in arable soil microbial communities. Soil Biology and Biochemistry 33:1713-1722.

Hamilton, R., et al. 2015. Draft genomes of gammaproteobacterial methanotrophs isolated from terrestrial ecosystems. Genome Announcements 3:e00515-15.

Ho, A., E. van den Brink, A. Reim, S. Krause, and P. L. E. Bodelier. 2016a. Recurrence and frequency of disturbance have cumulative effect on methanotrophic activity, abundance, and community structure. Frontiers in Microbiology 6:1493.

Ho, A., C. Lüke, A. Reim, and P. Frenzel. 2016b. Resilience of (seed bank) aerobic methanotrophs and methanotrophic activity to desiccation and heat stress. Soil Biology and Biochemistry 101:130-138.

Hoefman, S., D. van der Ha, N. Boon, P. Vandamme, P. De Vos, and K. Heylen. 2014. Niche differentiation in nitrogen metabolism among methanotrophs within an operational taxonomic unit. BMC Microbiology 14:83.

Jousset, A., B. Schmid, S. Scheu, and N. Eisenhauer. 2011. Genotypic richness and dissimilarity opposingly affect ecosystem functioning. Ecology Letters 14:537-545.

Kalyuzhnaya, M. G., A. E. Lamb, T. L. McTaggart, I. Y. Oshkin, N. Shapiro, T. Woyke, and L. Chistoserdova. 2015. Draft genome sequences of gammaproteobacterial methanotrophs 
isolated from Lake Washington sediment. Genome Announcements 3:e00103-e00115.

Knief, C. 2015. Diversity and habitat preferences of cultivated and uncultivated aerobic methanotrophic bacteria evaluated based on pmo $A$ as molecular marker. Frontiers in Microbiology 6:1346.

Krause, S., P. M. Van Bodegom, W. K. Cornwell, and P. L. E. Bodelier. 2014a. Weak phylogenetic signal in physiological traits of methaneoxidizing bacteria. Journal of Evolutionary Biology 27:1240-1247.

Krause, S., X. Le Roux, P. A. Niklaus, P. M. Van Bodegom, J. T. Lennon, S. Bertilsson, H.-P. Grossart, L. Philippot, and P. L. E. Bodelier. 2014b. Trait-based approaches for understanding microbial biodiversity and ecosystem functioning. Frontiers in Microbiology 5:251.

Le Mer, J., and P. Roger. 2001. Production, oxidation, emission and consumption of methane by soils: A review. European Journal of Soil Biology 37:25-50.

Levine, U. Y., T. K. Teal, G. P. Robertson, and T. M. Schmidt. 2011. Agriculture's impact on microbial diversity and associated fluxes of carbon dioxide and methane. ISME Journal 5:1683-1691.

Lindner, A. S., A. Pacheco, H. C. Aldrich, A. C. Staniec, I. Uz, and D. J. Hodson. 2007. Methylocystis hirsuta sp. nov., a novel methanotroph isolated from a groundwater aquifer. International Journal of Systematic and Evolutionary Microbiology 57: 1891-1900.

Loreau, M., and A. Hector. 2001. Partitioning selection and complementarity in biodiversity experiments. Nature 412:72-76.

Lüke, C., and P. Frenzel. 2011. Potential of pmoA amplicon pyrosequencing for methanotroph diversity studies. Applied and Environmental Microbiology 77:6305-6309.

Maron, J. L., M. Marler, J. N. Klironomos, and C. C. Cleveland. 2011. Soil fungal pathogens and the relationship between plant diversity and productivity. Ecology Letters 14:36-41.

Martin, M. 2011. Cutadapt removes adapter sequences from high-throughput sequencing reads. EMBnet.journal 17:10-12.

Martiny, A. C., K. Treseder, and G. Pusch. 2013. Phylogenetic conservatism of functional traits in microorganisms. ISME Journal 7:830-838

McDonald, I. R., L. Bodrossy, Y. Chen, and J. C. Murrell. 2008. Molecular ecology techniques for the study of aerobic methanotrophs. Applied and Environmental Microbiology 74:1305-1315.

McWilliam, H., W. Li, M. Uludag, S. Squizzato, Y. M. Park, N. Buso, A. P. Cowley, and R. Lopez. 2013. Analysis tool web services from the EMBL-EBI. Nucleic Acids Research 41:W597-W600.

Mohanty, S. R., P. L. E. Bodelier, V. Floris, and R. Conrad. 2006. Differential effects of nitrogenous fertilizers on methane-consuming microbes in rice field and forest soils. Applied and Environmental Microbiology 72:1346-1354.

Nannipieri, P., J. Ascher, M. T. Ceccherini, L. Landi, G. Pietramellar, and G. Renell. 2003. Microbial diversity and soil functions. European Journal of Soil Science 54:655-670.

Niklaus, P. A., J. Alphei, D. Ebersberger, D. Kandeler, C. Kampichler, and D. Tscherko. 2003. Six years of in situ $\mathrm{CO}_{2}$ enrichment evoke changes in soil structure and soil biota of nutrient-poor grassland. Global Change Biology 9:585-600.

Petchey, O. L., and K. J. Gaston. 2006. Functional diversity: Back to basics and looking forward. Ecology Letters 9:741-758.

Philippot, L., A. Spor, C. Hénault, D. Bru, F. Bizouard, C. M. Jones, A. Sarr, and P.-A. Maron. 2013. Loss in microbial diversity affects nitrogen cycling in soil. ISME Journal 7:1609-1619.

R Core Team. 2013. R 3.0. R Project for Statistical Computing, Vienna, Austria. www.r-project.org

Replansky, T., and G. Bell. 2009. The relationship between environmental complexity, species diversity and productivity in a natural reconstructed yeast community. Oikos 118:233-239.
Salles, J. F., F. Poly, B. Schmid, and X. Le Roux. 2009. Community niche predicts the functioning of denitrifying bacterial assemblages. Ecology 90:3324-3332.

Salles, J. F., X. Le Roux, and F. Poly. 2012. Relating phylogenetic and functional diversity among denitrifiers and quantifying their capacity to predict community functioning. Frontiers in Microbiology 3:1-15.

Schmid, B., M. Baruffol, Z. Wang, and P. A. Niklaus. 2017. A guide to analyzing biodiversity experiments. Journal of Plant Ecology 10:91-110

Seghers, D., K. Verthé, D. Reheul, R. Bulcke, S. D. Siciliano, W. Verstraete, and E. M. Top. 2003. Effect of long-term herbicide applications on the bacterial community structure and function in an agricultural soil. FEMS Microbiology Ecology 46:139-146.

Serrano-Silva, N., C. Valenzuela-Encinas, R. Marsch, L. Dendooven, and R. J. Alcántara-Hernández. 2014. Changes in methane oxidation activity and methanotrophic community composition in saline alkaline soils. Extremophiles 18:561-571.

Siljanen, H. M. P., A. Saari, S. Krause, A. Lensu, G. C. J. Abell, L. Bodrossy, P. L. E. Bodelier, and P. J. Martikainen. 2011. Hydrology is reflected in the functioning and community composition of methanotrophs in the littoral wetland of a boreal lake. FEMS Microbiology Ecology 75:430-445.

Spehn, E. M., et al. 2002. The role of legumes as a component of biodiversity in a cross-European study of grassland biomass nitrogen. Oikos 98:205-218.

Stein, L. Y., et al. 2010. Genome sequence of the obligate methanotroph Methylosinus trichosporium strain OB3b. Journal of Bacteriology 192:6497-6498.

Tillett, D., and B. Neilan. 2000. Xanthogenate nucleic acid isolation from cultured and environmental cyanobacteria. Journal of Phycology 36:251-258.

Tilman, D. 1997. Distinguishing the effects of species diversity and species composition. Oikos 80:185.

Tilman, D., D. Wedin, and J. Knops. 1996. Productivity and sustainability influenced by biodiversity in grassland ecosystems. Nature 379:718-720.

Ward, N., et al. 2004. Genomic insights into methanotrophy: The complete genome sequence of Methylococcus capsulatus (Bath). PLoS Biology 2:e303.

Wartiainen, I., A. G. Hestnes, I. R. McDonald, and M. M. Svenning. 2006a. Methylocystis rosea sp. nov., a novel methanotrophic bacterium from Arctic wetland soil, Svalbard, Norway $\left(78^{\circ} \mathrm{N}\right)$. International Journal of Systematic and Evolutionary Microbiology 56:541-547.

Wartiainen, I., A. G. Hestnes, I. R. McDonald, and M. M. Svenning. 2006b. Methylobacter tundripaludum sp. nov., a methane-oxidizing bacterium from Arctic wetland soil on the Svalbard islands, Norway $\left(78^{\circ} \mathrm{N}\right)$. International Journal of Systematic and Evolutionary Microbiology 56:109-113.

Wertz, S., V. Degrange, J. I. Prosser, F. Poly, C. Commeaux, T. Freitag, N. Guillaumaud, and X. Le Roux. 2006. Maintenance of soil functioning following erosion of microbial diversity. Environmental Microbiology 8:2162-2169.

Wertz, S., V. Degrange, J. I. Prosser, F. Poly, C. Commeaux, N. Guillaumaud, and X. Le Roux. 2007. Decline of soil microbial diversity does not influence the resistance and resilience of key soil microbial functional groups following a model disturbance. Environmental Microbiology 9:2211-2219.

Whittenbury, R., K. C. Phillips, and J. F. Wilkinson. 1970. Enrichment, isolation and some properties of methane-utilizing bacteria. Journal of General Microbiology 61:205-218.

Wohl, D. L., S. Arora, and J. R. Gladstone. 2004. Functional redundancy supports biodiversity and ecosystem function in a closed and constant environment. Ecology 85:1534-1540. 